\title{
4 Ausblick aus den Stufen auf Plessners Gesamtwerk und die aktuelle Diskussionslage
}

Nach der Lektüre der Stufen und ihres systematischen Kommentars kann es von Interesse sein, die aufgezeigte Problematik in bestimmten Hinsichten weiterzuverfolgen. Daher erinnere ich in dem ersten folgenden Schritt an die Stellung dieses Buches in dem Gesamtwerk Plessners. Im zweiten Schritt verweise ich auf die aktuelle Diskussion über dieses Standardwerk, die seine genauere Lektüre vertiefen könnte. Schließlich deute ich im dritten Schritt exemplarisch an, wie diese Diskussion in die gegenwärtigen systematischen Bemühungen um eine Philosophische Anthropologie im weiten Sinne geöffnet werden kann, ganz gleich, ob in ihnen bereits mit Plessner gearbeitet wird oder noch nicht.

\subsection{Einordnung der Stufen in das Gesamtwerk Plessners}

Schaut man zurück in den Arbeitsplan von Plessner am Anfang der Stufen, wo stehen wir nun an ihrem Ende? Als den Zweck oder das Ziel seines Unternehmens gibt er die „Neuschöpfung der Philosophie unter dem Aspekt der Begründung der Lebenserfahrung in Kulturwissenschaft und Weltgeschichte (30) ${ }^{1}$ an. Als Etappen auf dem Wege zu diesem Ziel nennt er die „Grundlegung der Geisteswissenschaften durch Hermeneutik, Konstituierung der Hermeneutik als philosophische Anthropologie, Durchführung der Anthropologie auf Grund einer Philosophie des lebendigen Daseins und seiner natürlichen Horizonte“ (ebd.). Der Aspekt, unter dem der Weg zu diesem Zweck beschritten wird, ist der des Lebens: „In seinem Mittelpunkt steht der Mensch. Nicht als Objekt einer Wissenschaft, nicht als Subjekt seines Bewusstseins, sondern als Objekt und Subjekt seines Lebens, d. h. so, wie er sich selbst Gegenstand und Zentrum ist“ (31). Als „personale Lebenseinheit“ gehe der Mensch nicht in dem Gegensatz zwischen Physischem und Psychischem auf, sondern stelle er deren Einheit auf eine Weise dar, die diesem exklusiven Dualismus gegenüber „neutral“ oder „,indifferent“ sein kann (32). Gibt es in diesem personalen Leben nicht nur Zufall, sondern auch eine „Wesenskoexistenz“, d. h. einen „strukturgesetzlichen Zusammenhang“ zwischen dem Lebenshorizont als Welt und der Personalität

1 Alle in diesem Kapitel in Klammern gesetzte Seitenzahlen beziehen sich auf Helmuth Plessner (1975): Die Stufen des Organischen und der Mensch. Einleitung in die philosophische Anthropologie [1928], Berlin/New York. 
als Lebenseinheit? Diese Frage soll „horizontal“ im Hinblick auf das „SubjektObjekt der Kultur“ und „vertikal“ hinsichtlich des „Subjekt-Objekts der Natur“ (ebd.) untersucht werden. Um den Zugang zur „Qualität“ der Lebenserfahrung, also Anschauung zu gewinnen, wird die phänomenologische Deskription als „ein wesentliches Mittel (nicht das einzige)“ (30) verwendet.

Was Plessner nun am Ende der Stufen erreicht hat, ist in vertikaler Richtung eine Naturphilosophie des lebendigen Daseins, die Lebendiges von Unbelebtem und unter dem Lebendigen verschiedene Organisations- und Positionalitätsformen in dem einheitlichen Zusammenhang der Natur qualitativ zu unterscheiden vermag. Diese Naturphilosophie deduziert nicht aus dem anschaubaren Sachverhalt, sondern im Hinblick auf die Verwirklichung des anschaubaren Sachverhaltes, wie einem lebendigen Körper seine Grenze angehört und sie von ihm vollzogen wird, die Bedingungen dafür, unter denen der angeschaute Sachverhalt wirklich sein kann (114f., 122). Die „Kategorien“, unter denen die Anschauung des Lebendigen in seiner Seinsweise und in seinem wirklichen Vollzug verstanden wird, stellen eine je spezifische Einheit von Anschauung und Denken des Lebendigen dar (65f.). Sie referieren auf „Lebenssphären“ der „Einheit von Subjekt und Objekt“ (66f., 244). Die theoretische Ordnung der Kategorien weist „mehr Verwandtschaft mit einer Dialektik als mit einer Phänomenologie“ (115, vgl. auch 34, 73, 113) auf, ohne des positiven Absoluten zu bedürfen (150f., 305), das durch vorschnelle Verallgemeinerung und Verselbständigung des Dritten eines Gegensatzes zum „Generalnenner“ (152) allen Seins entsteht. Die theoretische Ordnung der Kategorien in „Stufen“, die sich auseinander ergeben, legt die struktur-funktionale Pluralität der Lebensphänomene im Rahmen ihrer Einheit, als „Manifestation des Grundsachverhaltes“ der Positionalität (115), frei. Bei dieser Naturphilosophie handelt sich um eine solche hermeneutische Ontologie für die Naturphänomene des Lebendigen, die - gemessen an Kant und Hegel - „quasi-transzendental“ und „quasidialektisch“ (Krüger 2006a) verfährt, indem sie den Fokus des Philosophierens vom selbstbewussten Charakter des Wissens in die Personalität der Lebensführung verlegt.

Im Schlusskapitel über die Sphäre personalen Lebens geht diese Naturphilosophie zur Konstituierung der „allgemeinen Hermeneutik“ als „philosophische Anthropologie“ (28) über. In den drei anthropologischen Grundgesetzen werden jene strukturgesetzlichen Zusammenhänge expliziert, nach denen eine Wesenskoexistenz von Welt und personalem Leben in der Natur wirklich sein kann. Damit holt die „Rückkehr zum Objekt“ (31) oder die „Wendung zum Objekt“ (72) der Natur in dieser Natur selbst die Subjekt-Objekt-Einheit personalen Lebens ein, nämlich so, dass die Natur „mit dem persönlichen Leben in selber 
Höhe“ liegt (27). Dies eröffnet ein neues, weder reduktiv monistisches noch dualistisches Naturverständnis, nach dem der exzentrische Bruch mit der zentrisch positionierten Natur auf geistig-kulturelle und soziale Weise zur Lebenseinheit vollzogen werden kann, also als das Subjekt-Objekt der Soziokultur. In der geschichtlichen Wirklichkeit gibt es keine Trennung von Natur und Kultur, wie sie unter dualistischem Vorzeichen in der Trennung von Natur- und Geisteswissenschaften eingerichtet worden ist, sondern ihren Zusammenhang. Deshalb verweist Plessner im zweiten anthropologischen Grundgesetz auf seine Einheit der Sinne. Eine Ästhesiologie des Geistes (1923), in der die kulturellen Lebenshaltungen von Personen rekonstruiert werden, in denen Gegenstände und Personen angeschaut und gedeutet, kundgegeben und verstanden werden können, indem eine symbolisch funktionale Integration der verschiedenen Sinnesmodalitäten durch Personen vollzogen wird (14, 24, 35, 332, 340). Daher verweist er auch im dritten anthropologischen Grundgesetz auf die soziale Verwirklichung der Mitwelt für die ontisch-ontologisch zweideutige Individualisierung von Personalität in Gemeinschafts- und Gesellschaftsformen, die er in den Grenzen der Gemeinschaft (1924) entworfen hatte (335, 344f.). Das SubjektObjekt der Natur ist nur unter der Voraussetzung des Subjekt-Objekts der Soziokultur lebenswirklich, wie auch das Umgekehrte gilt: Die geistig-kulturellen Leistungen von Personen werden soziokulturell durch Rollen für Körperleiber ermöglicht und integrieren die körper-leiblichen Sinnesmodalitäten der sie ausübenden Lebewesen.

Im Schlusskapitel der Stufen wird zwar den Kategorien gemäß der Zusammenhang von Natur und Kultur in der exzentrischen Positionalität als wirkliche Ermöglichung begriffen, eben in den anthropologischen Grundgesetzen der natürlichen Künstlichkeit, vermittelten Unmittelbarkeit und des utopischen Standortes, aber diese Kategorien der Verwirklichung exzentrischer Positionalität und ihrer Grenzen werden noch durch die primär naturphilosophische Rekonstruktion der der personalen Lebenssphäre nötigen Ermöglichungsstrukturen gewonnen. Die mitgeführten Hinweise und Verweise auf dafür spezifische Phänomene, die es anzuschauen und zu verstehen gelte, können nicht in diesem vertikalen Teilprojekt ausgeführt werden. Wenn man sich fragt, an welchem Phänomenbestand die exzentrische Positionalität angeschaut und verstanden werden kann, und zwar so, dass der geschichtliche Zusammenhang zwischen Natur und Kultur auf gleicher Augenhöhe hervortritt, dann sollte man sich Plessners späterem Buch Lachen und Weinen. Eine Untersuchung der Grenzen menschlichen Verhaltens (1941) zuwenden, was wir hier im 2. Kapitel getan haben. Verlieren Personen in Situationen ihre Selbstbeherrschung, gemessen an den Maßstäben ihrer Soziokultur und ihrer Individualisierung, so fallen sie 
durch Mehrsinnigkeit ins Lachen und durch Sinnverlust ins Weinen. Dadurch tritt das Auseinanderfallen von Körperhaben und Leibsein hervor, die ansonsten durch den Vollzug der Person verschränkt werden. Die Personalität wird zwischen Lachen und Weinen erlernt, indem man mit Anderen mitmacht, sie nachmacht und schließlich Sachverhalte und Personen nachahmt, also ein Was und ein Wer nachahmt, das sich ablösen lässt vom Mantel seiner Eigenschaften in Raum und Zeit (Krüger 2014b, vgl. hier 10. Kapitel). Die Exzentrierung der Person von ihrem Körperleib wird in der Übernahme der Rolle anderer Personen erlernt, wofür die Schauspielerei (Plessner 1982i) das entfaltete Modell darstellt (Krüger 1999, 4. u. 5. Kap.; Zweiter Teil des vorliegenden Buchs).

Was nun aber am Ende der Stufen als das Wichtigste noch immer aussteht, sind nicht die nächsten, sondern die übernächsten Etappen auf dem Wege zum Ziel, d. h. zu der „Neuschöpfung der Philosophie unter dem Aspekt einer Begründung der Lebenserfahrung in Kulturwissenschaft und Weltgeschichte“ (30). Plessner hatte zwar einen kultur- und sozialphilosophischen Zugang zu der horizontalen Frage nach dem Subjekt-Objekt in der Soziokultur entworfen und jetzt in den Stufen auch einen naturphilosophischen Zugang zu der vertikalen Frage nach dem Subjekt-Objekt in der Natur vorgelegt, aber das geschichtsphilosophische Projekt stand für die Einlösung des Arbeitsplans im Ganzen noch bevor. Dafür waren die bisher entworfenen strukturgesetzlichen Zusammenhänge für die Ermöglichung von personaler Soziokultur überhaupt noch zu statisch, noch zu wenig aus der Dynamik in Geschichtsprozessen selber entwickelt worden. Plessner schloss sich zwar grundsätzlich schon in den Stufen Georg Mischs Systematisierung von Wilhelm Diltheys Philosophie des geschichtlichen Lebens (19-25) an, aber 1927 hatte Heidegger in Sein und Zeit bereits einen Entwurf dafür vorgelegt, wie man von der Existenzialität des Daseins im Horizont des Sinns von Sein eine hermeneutische Wende der Phänomenologie in die Zeitlichkeit vornehmen kann. Es bedurfte noch einiger Jahre des $\mathrm{Zu}$ sammenwirkens mit Georg Misch (Schürmann 2011, 4. u. 5. Kap.), der seine Monographie Lebensphilosophie und Phänomenologie. Eine Auseinandersetzung der Dilthey'schen Richtung mit Heidegger und Husserl (1930) zunächst als Artikelserie in Plessners Zeitschrift Der philosophische Anzeiger publizierte (192930), bis Plessner seine geschichtsphilosophischen Studien Macht und menschliche Natur. Ein Versuch zur Anthropologie der geschichtlichen Weltansicht (Plessner 1981d, 1931) und Das Schicksal deutschen Geistes im Ausgang seiner bürgerlichen Epoche (Plessner 1982e, 1935, ab 1959 unter dem Titel Die verspätete Nation. Über die politische Verführbarkeit bürgerlichen Geistes) anschließen konnte (Krüger 1999, 6. Kap.; Mitscherlich 2007, 3. Kap.; Krüger 2013a). 
1937 zieht Plessner eine vorläufige Bilanz seines Gesamtprojekts, die dann wirkungsgeschichtlich nach dem Zweiten Weltkrieg auch entscheidend wurde. In seinem Groninger Exil hielt Plessner seine Antrittsvorlesung über Die Aufgabe der Philosophischen Anthropologie (Plessner 1983d), in der er sein Vorgehen wie folgt zusammenfasst: Im Unterschied $\mathrm{zu}$ der gleichnamigen philosophischen Subdisziplin spricht seine Philosophische Anthropologie erstens theoretisch den Menschen „als Menschen“ an: Dieser hermeneutische Anspruch verlässt sich weder auf eine zoologische „Spezies Homo sapiens“ noch beruht er auf der Exklusion Anderer durch die eigene Religion, Kultur oder das eigene Volk.

In der Philosophischen Anthropologie ist der Mensch als Mensch angesprochen und in diesem Zusatz eine Einschränkung auf den Bereich vorgenommen, der zwischen den Extremen größtmöglicher Vereinzelung und größtmöglicher Verallgemeinerung eine nicht genau festzulegende Mitte hält. (Plessner 1983d, 36)

Die wirkliche Mitte, in der sich die „Wesensverfassung“ zwischen Einzelnem und Allgemeinem im Menschsein vollzieht, wird selbst zur theoretischen Forschungsaufgabe, statt sie für entweder eine einzige Vereinzelung (die individuell je unvertretbare Existenz) oder eine einzige Verallgemeinerung (als Spezies, Gesellschaft, Kultur, Sprache, Vernunft) des Menschseins von vornherein auszuschließen.

Zweitens bedeutet der Zusatz ,als Mensch“ einen praktischen Anspruch, für dessen Erfüllung ebenso wenig allgemein anerkannte Garantien gegeben werden können. Dass wir Menschen sind und sein sollen, diese Entdeckung oder diese Forderung verdanken wir einer bestimmten Geschichte, der griechischen Antike und der jüdisch-christlichen Religiosität. (ebd., 37)

Weil wir inzwischen aus historischer Erfahrung,

[...] durch die Kritik der Entwicklungsidee, durch die politische und ideologische Bekämpfbarkeit der humanitas um die Gewagtheit und Rückhaltlosigkeit des ,Menschen“Gedankens wissen, müssen wir das Menschsein in der denkbar größten Fülle an Möglichkeiten, in seiner unbeherrschbaren Vieldeutigkeit und realen Gefährdetheit so zum Ansatz bringen, dass die Gewagtheit eines derartigen Begriffes als Übernahme einer besonderen Verantwortung vor der Geschichte verständlich wird. (Ebd.)

Die Philosophische Anthropologie geht diese theoretisch-praktische Aufgabe an, indem sie drei Grundsätzen im Hinblick auf eine dreifache Verbindung folgt, eine Verbindung nicht nur zur Philosophie, sondern auch zu den Einzelwissenschaften und zur geschichtlichen Situation. Erstens gelte in der Philosophischen Anthropologie „die methodische Gleichwertigkeit aller Aspekte, in 
denen menschliches Sein und Tun sich offenbart“, darunter des physischen, psychischen, geistig-sittlichen und religiösen Aspekts, „für die sogenannte Wesenserkenntnis vom Menschen“ (ebd., 39). Diese methodische Gleichwertigkeit richtet sich gegen alle „materialistischen, idealistischen, existentialistischen Einseitigkeiten“ (ebd., 38). Zweitens gehe es um die Art und Weise der Einheit dieser Aspekte und damit des Überganges zwischen ihnen. Diese Art und Weise von Einheit sei von derselben „Ursprünglichkeit, wie sie der Mensch in seinem Geschichte werdenden Dasein beweist, in dem er sie sich erringt“ (ebd., 39). Es handelt sich also um eine qualitative Einheit der Aspekte, die in dem phänomenologischen Sinne „ursprünglich“ ist, dass sie nicht aus der Erkenntnis anderer Sachverhalte abgeleitet werden kann. Sie ist lebensweltlich durch keine „isolierende Methode des Laboratoriums“ (ebd., 47) zur Darstellung und Messung einzelwissenschaftlicher Erkenntnisse zu ersetzen. Diese qualitative Einheit ist aber auch nicht unbefragbar vorgegeben, sondern aus dem in der Geschichte werdenden Dasein errungen, also hermeneutisch zu erschließen. Ihre Veränderung kann besser oder schlechter ausfallen, was zu beurteilen eine „positive Zusammenarbeit“ mit den „Natur- und Kulturwissenschaften vom Menschen“ erfordert: Plessner gibt das Beispiel von der Erkenntnis „der Wirkungseinheit der Person zwischen Leib und Körper, Körper und Geist“ im biomedizinischen Kontext zwischen Arzt und Patient, wodurch auch die „Erkenntnistheorie und Ontologie vor neue Aufgaben“ (ebd., 48) gestellt werden (siehe im vorliegenden Band 5. Kapitel). Der dritte Grundsatz bezieht sich auf die geschichtliche Situation, in der die überweltlichen Autoritäten zerfallen sind und die innerweltlichen Autoritäten angesichts von Individualisierung und Pluralisierung strittig bleiben (ebd., 41 mit Verweis auf sein Buch Verspätete Nation). Er „,bestimmt die Funktion der Philosophischen Anthropologie, die sich ihrer theoretischen Grenzen im Hinblick auf ihre praktische Verantwortung gegen die Unergründlichkeit des Menschenmöglichen bewusst ist“" (ebd., 39 mit Verweis auf Plessner 1981d, 44). Daher können die Strukturformeln für die Erkenntnis der Wesensverfassung „keinen abschließend-theoretischen, sondern nur einen aufschließend-exponierenden Wert“ beanspruchen: In dieser Sicherung der Unergründlichkeit des Menschen komme „der Ernst der Verantwortung vor allen Möglichkeiten“ zum Ausdruck, in denen sich der Mensch „verstehen und also sein kann“ (Plessner 1983d, 39).

Nach der phänomenologischen und hermeneutischen Methode bringt Plessner erneut auch eine bestimmte Art und Weise von Dialektik zur Sprache, die zweifellos auf Hegels Phänomenologie des Geistes anspielt, ohne Hegels systematisch positive Fassung des Absoluten als Geist Gottes zu beanspruchen. Die moderne Skepsis gegen jede Autorität rechne mit einer Selbsttäuschung, die 
durch ihre reflexive Rückführung auf ein bestimmtes Subjekt behoben werden könne, was sich von Kant über Marx und Nietzsche bis Freud oft genug wiederholt hat. Diese Skepsis sei auch gegen die „Selbstvergötterung des Menschen“ im Namen der „Fortschritte“ an „Verfügungsgewalt“ durch Wissenschaft und Technik zu richten (ebd., 50), womit Plessners Kritik immanent einsetzt. Aber die Verwirklichung dieser Skepsis überwinde sie auch, denn sie bringe Anderes zum Vorschein, als in der dualistischen Skepsisart an alleiniger Selbsttäuschung eines Subjekts erwartet werde. Die Verwirklichung dieser Skepsis lasse die „Grenze“ klarwerden, „bis zu der sich der Mensch als Mensch in Frage stellen kann“: Ohne ihre Verwirklichung wird man „den Verdacht gegen das Recht, vom Menschen als einem besonderen und auf seinen Gattungscharakter verpflichteten Wesen zu sprechen, nicht loswerden“ (ebd., 45). Der durch seine „Destruktion“ führende Begriff des „Menschen“ habe „Wagnischarakter“ und erfordere den „Mut zur rückhaltlosen Skepsis als einer Methode des Menschen, sich durch Selbstentsicherung wiederzufinden“ (ebd., 37 u. 46) So heißt es denn zusammenfassend kursiv: „Die im Sinne ihrer Überwindung verwirklichte Skepsis ist allein möglich als Philosophische Anthropologie“ (ebd., 41).

Es kann hier nicht nebenbei die breite und tiefe Diskussion über den $\mathrm{Zu}-$ sammenhang zwischen Plessners Natur- und Geschichtsphilosophie ausgetragen werden. Der Konsens sollte aber m. E. zumindest darin bestehen, dass nicht einmal der Arbeitsplan der Stufen ohne die geschichtsphilosophischen Studien als erfüllt angesehen werden könnte. Das Programm der Stufen geht von Anfang an deutlich über den in ihnen geplanten Teil einer philosophischen Biologie hinaus. Umso mehr gilt für diejenigen Autorinnen und Autoren, die die Gleichrangigkeit der Geschichts- mit der Naturphilosophie in einer insgesamt gebrochenen Lebensphilosophie Plessners (Mitscherlich 2007) oder den hermeneutischen Primat der geschichtsphilosophischen Verwirklichung des Soziokulturellen (Lindemann 2009 u. 2014; Schürmann 1997, 2011 u. 2014; Wunsch 2014) vertreten, dass die Stufen vom Standpunkt der in Macht und menschliche Natur explizierten Hermeneutik her einzuordnen sind. Unter dem Primat der Hermeneutik wurde Plessners Philosophische Anthropologie von vielen wichtigen Autoren seit langem explizit gelesen (Bollnow 1956; Habermas 1958, Honneth/Joas 1980; Schnädelbach 1983).

\subsection{Zur aktuellen Diskussion über die Stufen}

Beaufort hat die jüngere Diskussion über die Stufen damit eröffnet, dass er das naturkonstitutionstheoretische Verfahren der Stufen vom Standpunkt der gesellschaftlichen Konstruktion der Natur aus kritisierte (Beaufort 2000). Ich habe 
in meiner Einführung diese Diskussion im Überblick dargestellt (1.7.), darunter insbesondere auf Mitscherlichs Herausarbeitung der „doppelseitigen Deduktion“ (Mitscherlich 2007) und auf Grenes Herausstellung der naturphilosophischen Originalität von Plessners Stufen verwiesen (1. 2.). Wunsch fasst die gegenwärtige Diskussion dahingehend zusammen, dass die exzentrische Positionalität ein Prinzip der Ansprechbarkeit als Mensch bzw. Person darstelle, nicht aber als ein Prinzip der Konstitution im Sinne empirischer Kriterien von Gegenständen zu verstehen ist. Zudem trete in Macht und menschliche Natur das ethische Prinzip von der Verbindlichkeit der Unergründlichkeit explizit hinzu, das zu politischer Orientierung entfaltet werde. Das Prinzip der Ansprechbarkeit und das der Verbindlichkeit von Unergründlichkeit begrenzten sich gegenseitig (Wunsch 2014, 231-235, 237f., 258-264).

Eine Ausnahme in der gegenwärtigen Diskussion über die systematischen Aufgaben in Plessners Philosophischer Anthropologie stellt Fischer dar, der Plessner von der Wirkungsgeschichte des Ausdrucks „Philosophische Anthropologie“ im Werk von Arnold Gehlen her liest. Bei Gehlen liegt indessen (auch laut dessen Selbstauskünften) überhaupt keine phänomenologische und dialektische Hermeneutik anthropologischer Fragen vor, sondern eine „Philosophie“, die „Empirien“ zum „Thema“ Mensch „integriert“ (Gehlen 1986a 142f.). Dabei steht diese Integration unter der leitenden Frage, wie es der Mensch, der kein Tier mehr ist, dennoch schaffen kann, sein evolutionäres Überleben zu sichern: „wie ist es einem instinktentbundenen, dabei aber antriebsüberschüssigen, umweltbefreiten und weltoffenen Wesen möglich, sein Dasein zu stabilisieren?“ (Gehlen 1986b, 42). Und die Antwort lautet, da der Mensch nicht wie Tiere über spezialisierte Instinkte zur Anpassung an eine spezielle Umwelt verfügt: Alle vom Menschen gestalteten Produkte müssten „sekundär den primär versagten Automatismus des Verhaltens gestatten, und damit vor allem: die nicht selbstverständliche Regelmäßigkeit des Verhaltens gegenüber den Dingen und gegeneinander“ (ebd.). „Alles gesellschaftliche Handeln wird nur durch Institutionen hindurch effektiv, auf Dauer gestellt, normierbar, quasi-automatisch und voraussehbar“ (ebd.). Da im Falle des Menschen nicht mehr die Biologie den biologischen Maßstab des evolutionären Überlebens erfüllen kann, müsse die Sozial- und Kulturanthropologie das funktionale Äquivalent dafür leisten. Fischer vertritt daher funktional gesehen im Anschluss an Gehlen auch für die Plessner-Lektüre einen Primat der vertikalen Biophilosophie über die horizontale Kulturphilosophie, wobei die Geschichtsphilosophie bei ihm überhaupt nicht vorkommt (Fischer 2008, 549).

Demgegenüber betont auch Peter Sloterdijk, der 2017 den Helmuth Plessner-Preis der Stadt Wiesbaden erhalten hat, den Gegensatz zwischen 
Gehlens und Plessners Konzeptionen. Während Gehlen noch ein Denker des vertikalen Mangels im Menschen - gemessen am Tier - sei und damit der alten Anthro-Pathologie angehöre, bilde Plessners Werk eine Zäsur, denn es eröffne die vertikale Weite derjenigen ontologischen Aussteiger, die in einer überschüssigen exzentrischen Positionalität leben (Sloterdijk 2017). Daher hatte ich früher von der Aussetzung der lebendigen Natur als geschichtlicher Aufgabe in ihr (Krüger 2004b), von dem Schauspiel der Kultur im Spiel der Natur, darunter im Spiel zwischen Leibsein und Körperhaben, gesprochen (Krüger 2000a u. b). Ich hatte von Anfang an das Gesamtwerk Plessners unter dem geschichtsphilosophischen Prinzip der Unergründlichkeit, d. h. gemäß der Öffnung der Frage in die Zukunft des Ganzen personalen Lebens, rekonstruiert, was sich mit dem Primat einer Philosophie der offenen Fraglichkeit personalen Lebens in der gebrochenen und daher geistoffenen Natur bestens verträgt (Krüger 1999, 30-32, 265-270; Krüger 2015). Man verwechsele diese Naturphilosophie nicht mit Gehlens philosophischer Biologie als dem funktionalen Maßstab für die Sozial- und Kulturanthropologie. Die exzentrisch gebrochene und geistoffene Natur ist etwas anderes als dieser Maßstab (vgl. dagegen Mitscherlich 2007, 13f.).

Die Fraglichkeit der personalen Lebenssphäre, bevor (logisch gesehen) sich personale Lebewesen etwas und jemanden fragen können, rührt aus dem exzentrischen Bruch mit Körpern (in Raumzeit) und Leibern (in Raum- und Zeithaftigkeit) in das Nichts (der Raumzeit) und das Nirgendwo, Nirgendwann (der Raum- und Zeithaftigkeit) her (vgl. oben 12. 1.). Alle Versuche, auf diese Fraglichkeit in der exzentrischen Positionalität durch eine positive Auswahl aus den Potentialitäten des Geistes der Mitwelt zu antworten, schließen diese Fraglichkeit nicht vollständig ab (Krüger 2006b u. c). Diese Fraglichkeit geht als die Gestelltheit in den Bruch nicht darin auf, sich dem Bruch zu stellen und seine Überbrückung zu vollziehen. Personale Lebewesen schaffen nicht diese Gestelltheit in den Bruch ab, sondern schaffen es, in dieser Gestelltheit in dem Bruch zum Stehen zu kommen, indem sie die Überbrückung in diese Gestelltheit vollziehen. Das sich dem Bruch Stellen und seine Überbrückung Vollziehen verwirklicht die exzentrische Positionalität geschichtlich, d. h. auch nie vollständig und ein für alle Mal, als ob die Fraglichkeit verschwände, die das geschichtliche Fragen und Antworten ermöglicht und erfordert (siehe oben 3. 2. 3. 4.). Schürmann spricht das Problem zwischen der „Gestelltheit“ und dem „Vollzug“ in der exzentrischen Positionalität an, in dem die Fraglichkeit besteht, weshalb Plessner die Gestelltheit und den Vollzug, sich in ihr stellen zu können, stets von Neuem in Sozial-, Kultur- und Geschichtsphilosophie ,verteilt“ (Schürmann 2014, 103f.). Es gibt zwar schon naturphilosophisch im Behar- 
ren gegen das Werden eine Art und Weise von Verteilung (134), aber erst die Verteilungsweise von Personenrollen und deren geschichtliche Verwirklichung in Gemeinschafts- und Gesellschaftsformen ermöglicht es dem konkreten Individuum, den Hiatus des Nichts im Vollzug wirklich überbrücken zu können.

Die naturphilosophische Einsicht in den Hiatus und seine Verschränkungsnot hat Plessner auch im Auge, als er im Februar 1928 seinem Freunde Josef König, der gerade von Heideggers Sein und Zeit wie viele Zeitgenossen begeistert ist und dann bis 1933 zu Heideggers Philosophie wechseln wird, versucht zu erklären, worin die Differenz seiner Philosophie zu der von Heidegger besteht. „Hier finde ich den eigentlich schwachen Punkt Heideggers, der noch an einen ausgezeichneten Weg (der Ontologie) glaubt in der Rückinterpretation der Frage auf den angeblich sich Nächsten: den Fragenden (als ob wir fragen könnten, wenn wir nicht gefragt wären!).“ (König/Plessner 1994, 176, so auch V) Dieses Gefragtsein lässt sich nicht in diejenigen Frage-Antwort-Relationen auflösen, die Personen geschichtlich durch Zuordnungen einrichten, weshalb diese Einrichtungen geschichtlich veränderlich bleiben. Das „Gefragtsein jeder gestellten Frage“ ist „eine andere Verklammerung als die von Subjekt und Objekt, NoesisNoema etc.“ (König/Plessner 1994, 177). Die von Plessner freigelegten „sphärischen Strukturen“ sind „gegen den Gegensatz von Existenz und Sein, Zuhandenheit und Vorhandenheit, Subjekt und Objekt, Innen und Außen gleichgültig“ (ebd.). Sie erfüllen den Sinn dessen, „,was ohne wissenschaftliche Restriktion von uns natura sive mundus genannt wird“ (ebd.). Man dürfe dasjenige Schaffen, das das personale Leben auszeichnet, um sich seinem Gestelltsein in den Bruch der Natur gewachsen zu zeigen, nicht übertragen auf die Natur - in einer ganz anderen „Weite dessen, was hier Natur bedeutet“ (ebd.) -, als könne sie nichts Anderes sein denn die vom Menschen oder von (seiner idealen Verlängerung) Gott geschaffene Welt. „Darin sehe ich mein eigentliches philosophisches Ziel: Ersetzung des apokalyptischen Weltbegriffs, d.h. desjenigen Begriffs, der Welt als der Möglichkeit nach ens creatum fasst“ (ebd. 179). Daran gemessen stehe Heidegger noch im Banne der alten Tradition des Subjektivismus (V), der in der Moderne zu der oben genannten „Selbstvergötterung“ des Menschen führt und damit die nächste Apokalypse heraufbeschwört.

Heute ist seit Jahrzehnten die ökologische Problematik als Folgenakkumulation der Selbstermächtigung der Moderne zur Beherrschung der Natur bekannt und unter dem Titel des Anthropozäns ausgerufen worden. Noch immer soll die Natur nur Umwelt sein, die dem ontologischen Zentrum des je eigenen Selbstseins zu dienen hat, letzteres möge nur besser seine langfristigen statt kurzfristigen Interessen berücksichtigen. Natur auf gleicher Augenhöhe mit dem Geiste, wie bei Plessner, darf es so noch immer nicht geben (Block 2016), es 
sei denn negativ als Rache der Natur in einer Apokalypse (so ist die Natur noch immer anthropomorph gedacht, wie man sich auch Gott so vorgestellt hat) oder im Sinne einer Schickung des späten Heideggers. Heidegger hat in seiner Kehre das Sein gegen das Dasein verselbständigt, um auf diese Weise selber die Fraglichkeit vor (logisch) dem geschichtlichen Fragen und Antworten thematisieren zu können. In den letzten Jahren rührt sich auch wieder ein verstärktes Bedürfnis der Philosophie nach einem Realismus, der dem Konstruktivismus im Dienste des individuellen und kollektiven Selbstsein-Könnens Grenzen setzt. Aber oft wird dabei noch der neue Realismus an die Unmittelbarkeit der Erfahrung durch Konfusion von Merleau-Pontys Leib mit Heideggers Dasein (Dreyfus/ Taylor 2016) geknüpft, als ließe sich nicht mit Plessner die vermittelte Unmittelbarkeit (oben 3.2.) der Beziehungen in der exzentrischen Positionalität einsehen.

Dieses ökologische und jenes realistische Unterfangen sind nur zwei aktuelle Beispiele dafür, wie lohnenswert es sein kann, die Jahrzehnte anhaltende Kontroverse zwischen Plessner und Heidegger auf ihrem hohen Niveau verstehen zu lernen. Umso erfreulicher ist es, dass in jüngster Zeit die beiden großen Antipoden der deutschsprachigen Philosophien des 20. Jahrhunderts, eben Heideggers und Plessners Philosophien, endlich in eine Diskussion versetzt werden. In diese aktuelle Rekonstruktion werden inzwischen so viele Philosophien, Biologien und Medizin-Konzeptionen einbezogen, dass eine ganze diskursive Formation zur Philosophie der Lebensexistenzen im Hinblick auf ihre bio-medizinische Erforschung zum Vorschein kommt. Sie beinhaltet auch die philosophischen Positionen von Hans Driesch, Max Scheler, Ernst Cassirer, Georg Misch, Nicolai Hartmann, Karl Löwith, Hannah Arendt, Theodor W. Adorno, Max Horkheimer, Josef König (Schmitz 1996; Krüger 1999, 2001, 2009a, 2012, 2016; Damm/Gutmann/Manzei 2005; Schloßberger 2005; Großheim 2013; Schürmann 2011 u. 2014; Wunsch 2014), aber ebenso die Positionen führender Biologen und Mediziner von Karl Jaspers, Jakob von Uexküll über Jakobus Johannes Buytendijk und Viktor von Weizsäcker bis zu Adolf Portmann, den Gestaltpsychologen u. v. a. (Köchy/Michelini 2015; Danzer 2011 u. 2012). Plessners naturphilosophische Kombination aus Anschauung (qualitativer Erfahrung), Verstehen und dialektischer Krisis ist vom Grundansatz erneut fruchtbar, wenn man heutzutage insbesondere den Verstehensproblemen in der Exzentrierung und Rezentrierung personaler Lebewesen im Unterschied zu apersonalen Lebewesen nachgeht. Dies hat sich sowohl in der vergleichenden Hirn- und Verhaltensforschung gezeigt (Krüger 2010) als auch in dem Versuch, die Spezifik psychischer Krankheiten von personalen Lebewesen zu begreifen (Heinz 2014). Plessners hermeneutische Offenheit in der Interpretation 
der exzentrischen Positionalität, wodurch er den Zirkel des Menschseins kategorial überwunden hat, hat auch sozialphilosophische Früchte ermöglicht (Lindemann 2009 u. 2014).

\subsection{Zum Bedürfnis nach einer Philosophischen Anthropologie im weiten Sinne}

Folgt man der modernen Verwirklichung der exzentrischen Positionalität im Sinne der anthropologischen Grundgesetze (oben 3. Kap.), also hinsichtlich des wesentlichen Zusammenhanges zwischen personaler Lebensform und der sie ermöglichenden Welt, dann entstehen unter dem Aspekt der natürlichen Künstlichkeit anthropologische Fragen in den erwartbaren Grenzen dieser Verwirklichung. Es gilt dann nicht nur im ökologischen Verhältnis zur äußeren Natur die Grenzfrage zu beantworten, wie personale Lebensformen in die unbelebte und belebte Natur durch natürliche Künstlichkeit nachhaltig eingepasst werden können, darunter insbesondere das Verhältnis zu pflanzlichen und tierlichen Lebensformen zu gestalten ist. Es geht dann auch im Verhältnis zur eigenen Natur personalen Lebens durch natürliche Künstlichkeit um die Einrichtung von Grenzöffnungen und Grenzschließungen für die Zugehörigkeit zum Kreis der Personen, insbesondere am Lebensanfang und am Lebensende, und zur Ermöglichung eines guten Lebens für Personen. Schließlich folgen aus der technologischen Vergegenständlichung von natürlicher Künstlichkeit solche Fragen wie die nach dem Verhältnis künstlicher und kommunikativer Intelligenz zu dem personalen Geist in der personalen Lebenssphäre. Lindemann spricht von einem „Quadrat“ anthropologischer Fragen, über deren Lösung ein geschichtlicher Kampf zur Einrichtung von „Grenzregimen“ zwischen Öffnung und Schließung der jeweiligen Grenze entbrennt (Lindemann 2009, 83f., 123f., 126f.).

Unter dem Aspekt des zweiten anthropologischen Grundgesetzes der vermittelten Unmittelbarkeit kann man in der Tat erwarten, dass die moderne Verwirklichung der personalen Lebenssphäre generationenweise zu stets erneuten Wellen an Expressivität führt, statt je vollständig und für alle verbindlich nach einem bestimmten Maßstab durchrationalisiert werden zu können, wodurch der Konflikt zwischen Gemeinschaftsformen und Gesellschaftsformen erneut ausbricht und der Verschränkung bedarf (oben 3.3.). Auch dies führt geschichtlich zu vorläufigen Spektren zwischen der Schließung und Öffnung der Grenze, wer zum Kreis der Personen in der personalen Lebenssphäre grundsätzlich, vorrangig, nebenrangig etc. gehört. Unter dem Aspekt des dritten anthropologischen Grundgesetzes vom utopischen Standort lässt sich die stets erneute generatio- 
nenweise Herausbildung von Utopien erwarten, die exzentrische Lebewesen in religiösen und areligiösen Formen bewegen, ohne dass es zu einer endgültigen Überwindung dieser Pluralität von Formen durch eine einzige Form ein für alle Mal und für alle kommen kann.

Mir scheint, dass sich all diese kategorialen Erwartungen, die sich im Hinblick auf die moderne Verwirklichung der exzentrischen Positionalität aus Plessners Philosophischer Anthropologie ergeben, in den letzten Jahrzehnten von neuem bestätigt haben. Im heute allgemeinen, von Plessner ganz unabhängigen Sprachgebrauch erscheinen Fragen als anthropologische, wenn es um die Unterscheidung menschlicher Lebewesen von anderen Lebewesen oder Akteursarten innerhalb einer Gemeinsamkeit mit diesen geht. So wird die Frage gestellt, was uns Menschen denn von Affen oder intelligenten Robotern unterscheide, wobei die Intelligenz als Vergleichsmaßstab an Gemeinsamkeit vorausgesetzt wird. So berechtigt solche Fragen empirisch für bestimmte isolierende Kontexte sind, sie führen normative Fragen mit sich, da sie für die betroffenen Lebensformen als ganze praktisch relevant sind. In der weithin üblichen Arbeitsteilung zwischen empirischen und normativen Fragen entsteht so das Bedürfnis nach einer Integration zwischen den empirischen Fragen, die zusammengestellt einen anthropologischen Realitätskontakt halten mögen, und den philosophischen Fragen, die zu normativen Lösungen für die praktischen Probleme führen sollen. Wächst sich dieses Integrationsbedürfnis über einzelne anthropologische Fallgruppen hinausgehend aus, tendiert es dazu, eine philosophische Anthropologie zu werden. Dieses Bedürfnis nach einer philosophischen Anthropologie im weiten Sinne artikuliert sich in einem allgemein üblichen Sprachgebrauch, der terminologisch variabel ist und nicht an die spezielle, aber gleichnamige Strömung anschließen muss, die von Max Scheler und Helmuth Plessner begründet worden ist (Schnädelbach 1983). Die echten Sachfragen der Philosophischen Anthropologie im engeren Richtungssinne werden ihren Voraussagen gemäß expressiv variabel immer erneut geschichtlich artikuliert.

Dies sei - den Ausblick auf eine weitere Diskussion abschließend - an vier Beispielen verdeutlicht, die m. E. eine gewisse Repräsentativität beanspruchen dürfen. Plessners Stufen beginnen mit einer Konfrontation zwischen dem dualistischen Mainstream moderner Philosophie und der lebensphilosophischen Gegenströmung, um aus diesem Gegensatz zwischen einem einerseits Rationalität versprechenden Dualismus und andererseits einem intuitiven Monismus alles irrational Lebendigen durch Verschränkungen heraus zu gelangen. Je nachdem, in welche problemgeschichtlichen Bindungen die heute Philosophierenden zunächst kontingent hineinwachsen, entsteht das Grundproblem der 
Überwindung von entweder einem solchen Dualismus oder einem derartigen Monismus in innovativen Integrationsformen stets erneut. Man kann die Familienähnlichkeit der systematischen Problemlage leicht erkennen, ohne sich die lebensgeschichtlich kontingente Ausgangslage der betroffenen Philosophierenden schulpolitisch vorwerfen zu müssen.

Martha Nussbaum hatte früh einen neoaristotelischen Abstand zum neuzeitlichen Dualismus und seinen modernen Durchführungen, weshalb sie auf ihre Weise Max Schelers Thema von der Sinnordnung des personalen Gefühlslebens, d. h. von Liebe und Hass, von Scham und Ressentiment, von Mitfreude und Mitleid (Schloßberger 2005), wieder entdecken konnte, ohne dass Scheler für sie persönlich wichtig gewesen wäre (Nussbaum 2014). Stattdessen schloss sie problemgeschichtlich insbesondere an Michel Eyquem de Montaigne und Johann Gottfried Herder sowie systematisch an John Rawls Theorie der Gerechtigkeit an. Ihre philosophisch-anthropologische Integration zielt nicht mehr nur auf eine traditionelle Verteilungsgerechtigkeit der Dinge ab, sondern auf die Gerechtigkeit in den Chancen, dass Menschen die ihrer Gattung spezifischen Fähigkeiten (capabilities) ausbilden können. Hier liegt ein Problem des Überganges von normativ ermöglichenden Fragen und empirischen Fragen in beide Richtungen vor, zu dessen Lösung Plessner ein methodisch aufwendiges Verfahren im Zeichen des homo absconditus entwickelt hat, der Nussbaum systematisch fehlt.

Otfried Höffe hat demgegenüber Kant nicht wie üblich dualistisch gelesen, wodurch man erst durch Herder auf die Fragen einer philosophischen Anthropologie der Gemeinsamkeit alles Lebendigen und der geschichtlichen Vervollkommnung von Humanität kam (vgl. Krüger 2009a, 4. u. 9. Kap.). Höffe hat das ebenfalls bei Kant vorhandene Anliegen einer Integration von transzendentalen Ermöglichungsfragen mit den empirischen Fragen der anthropologischen Menschenkenntnis zu einer metaphysischen Wissenschaft entfaltet. Daraus erwächst eine zugleich transzendental und pragmatisch verfahrende philosophische Anthropologie, die Höffe insbesondere rechts- und staatsphilosophisch (Höffe 1999) sowie für das der Moderne konstitutive Thema der Freiheit (Höffe 2015) durchgeführt hat. Auch hier eröffnet sich ein interessantes Diskussionsfeld, wie genau die metaphysische Integration der transzendental ermöglichenden und der empirisch pragmatischen Fragen erfolgen kann (Krüger 2009a, 3. Kap.). Dazu lädt Plessners Verfahren der Kombination von phänomenologischer, geschichtlich-hermeneutischer und kategorial-dialektischer Methode in einer Metaphysik der Negativität des Absoluten (oben Kapitel 1.3.) ein.

Auch die hermeneutische Ontologie des Selbst- und Weltverständnisses von Charles Taylor hat auf die erneute Entdeckung der Human Condition zurückge- 
führt. Seine Rekonstruktion des geschichtlichen Wandels dieses Verständnisses während des letzten halben Jahrtausends hat nicht nur Plessners Abheben auf die Expressivität im weiten Sinne als den Motor geschichtlicher Veränderungen und darunter die moderne Stimme der Natur bestätigt (Taylor 1994), ohne Plessner näher gekannt zu haben. Taylor hatte früh durch Merleau-Pontys Leibesphänomenologie, die ihrerseits auch unter Einfluss Plessners entstanden war (siehe oben 1. Kap.), eine Distanz vom dualistischen Mainstream modernen Philosophierens gewonnen, die offen war für dasjenige, das er später das für Anderes und Fremdes „poröse“ Selbst im Unterschied zum gegenüber Anderem und Fremdem „abgepufferten“ Selbst nennen sollte (Taylor 2009, 79, 899). Vor allem aber steht seine Rekonstruktion im Einklang mit dem, was Plessner den utopischen Standort im weiten Sinne (oben 3.3.) nennt: Die Lage des Menschen im Ganzen bleibe durch allen geschichtlichen Wandel hindurch prekär und dilemmatisch, so sehr jede der traditionellen und reformerischen Religionsformen, der anti-humanistischen und humanistischen Bewegungen an die Erlösung des Menschen durch die jeweils eigene Utopie im Gegensatz zu den anderen glaube und dadurch auch das Gegenteil bewirke (ebenda 1058ff., 1116ff.). Taylor spricht von einer „tragischen Ironie“ in der conditio humana (ebenda 1156), die zu einem Vergleich mit denjenigen Grenzerfahrungen einlädt, die Plessner das gespielte und ungespielte Lachen und Weinen (Plessner 1982f) nannte. Was bei Taylor unter den Tendenzen, sich dieser Lage zu stellen, fehlt, ist die Option, Natur in sich gebrochen und daher geistoffen verstehen zu können, die sich in den klassischen Pragmatismen und der Philosophischen Anthropologie Plessners artikuliert hat (Krüger $2001 \mathrm{u}$. Krüger 2017e).

Schließlich steht das Werk von Michael Tomasello exemplarisch für die heutige transdisziplinäre Forschungslage einer evolutionären Anthropologie. Einerseits behandelt er die Frage, worin die Gemeinsamkeiten und Unterschiede zwischen Menschen und non-humanen Primaten, insbesondere Menschenaffen, bestehen. Diese Frage betrifft einen Teil der Fragerichtung, die Plessner die vertikale nannte. $\mathrm{Zu}$ ihr gehören auch die Gemeinsamkeiten und Unterschiede zwischen lebendigen und anorganischen Dingen, zwischen pflanzlichen und tierlichen Lebensformen, zwischen Primaten und anderen Säugetieren. Andererseits beschäftigt sich Tomasello mit der Frage der Humanontogenese in verschiedenen derzeitigen Kulturen, insbesondere anhand der Entwicklung von Kleinkindern, Vorschulkindern und jungen Schulkindern. Diese Frage betrifft einen Ausschnitt aus der Fragerichtung, die Plessner als die horizontale bezeichnete. Sie umfasst die Gemeinsamkeiten und Unterschiede von Lebensformen des Menschen im weltgeschichtlichen und gegenwärtigen Vergleich seiner Soziokulturen. Tomasello hat beide Ausschnitte aus der vertikalen und 
der horizontalen Untersuchungsrichtung in seinem Buch Origins of Human Communication (Tomasello 2008) zusammengeführt. Er hat inzwischen sowohl für die Naturgeschichte des menschlichen Denkens (Tomasello 2014a) und der menschlichen Moral (Tomasello 2016) als auch für die Humanontogese (Tomasello 2019) eine konzeptionelle Rekonstruktion vorgelegt. Wie war die Evolution unserer Vorfahren zum modernen Menschen und wie ist die moderne Ontogenese stets von neuem möglich? Um diese Fragen beantworten zu können, rekonstruiert er den Übergang von der individual intentionality zur joint intentionality und zur collective intentionality. Diese Unterscheidung zielt auf eine empirische Präzisierung für den Übergang von der zentrischen in die exzentrische Positionalität ab, den Plessner aber philosophisch im Ganzen anders fassen würde (Krüger 2014c u. 2016a). Plessner versteht die Transformation von Lebensformen kategorial durch die Personalität. Eine Person steht vertikal gesehen in der Differenz zwischen ihrem Körper-Haben und ihrem Leib-sein und horizontal betrachtet in ihrer Relation zu anderen Personen in der Mitwelt durch Rollen. Für diese Transformation durch inter-personale und intrapersonale Relationen in einer Mitwelt stellt die Unterscheidung von verschiedenen Formen der Intentionalität einen wichtigen, aber auch nur einen Aspekt dar. Dafür hat Tomasello im Jahre 2014 zu Recht den Helmuth Plessner-Preis der Stadt Wiesbaden, der Geburtsstadt Plessners, verliehen bekommen. 